\title{
On the detection of letters within redundant arrays
}

\author{
NEAL F. JOHNSON \\ The Ohio State University, Columbus, Ohio
}

\begin{abstract}
It has been reported (Johnson, 1977) that subjects can detect the presence or absence of a target letter (e.g., B) in a subsequent display more rapidly if the display consists of a single letter (e.g., $B$ or $L$ ) than if it is redundant in the sense of containing several instances of the same letter (e.g., BBBBB or LLLLL). Unfortunately, Krueger and Shapiro (1980) reported a series of similar experiments in which that effect was not obtained. The present study reports two experiments in which the original effect was obtained. In the first experiment, the single-letter displays were presented in one block of trials, and multiletter displays were presented in another block of trials, but the latter blocks contained a mixture of redundant (BBBBB) and nonredundant (BQTRZ) displays. To rule out the possibility that the effect was caused by some strategic carryover from the handling of the nonredundant displays to that of the redundant displays, in the second experiment those multiletter items were divided into separate blocks of redundant and nonredundant displays. While the advantage of single-letter displays over redundant displays was reduced somewhat, it was not eliminated.
\end{abstract}

When subjects are asked to detect whether a displayed word matches a predesignated target word, their response is faster than when their task is to detect whether the first letter of the word matches a predesignated target letter (Johnson, 1975, 1977, 1981), and this phenomenon has been referred to as the word priority effect (Sloboda, 1976, 1977). It has been proposed that this effect suggests that word processing may be in some sense holistic, at least to the extent that word-level information seems to be available for cognitive processing before letter-level information (Johnson, 1977, 1981, in press).

Although holistic processing can be operationally defined in terms of such differential availability of patternor word-level information, as opposed to component- or letter-level information, the problem is to provide a theoretical framework within which to understand how such empirical effects are obtained. One such framework is the pattern-unit model (Johnson, 1975, 1977, 1981), which has as its most basic assumption the hypothesis that perceivers always attempt to encode small visual patterns into memory by assigning the pattern a unitary cognitive representation, and that such unitary cognitive encoding does not entail a prior and comparable encoding of any of the pattern's components.

This model further assumes that direct component-level encoding could occur only if the attempts at pattern-level encoding have failed. For example, if the subjects' task was to identify a letter from within a presented word, this view would suppose that the word always would be encoded into memory as a unit, because such a pattern- or word-level code would be available. Given that occurred,

Address correspondence to Neal F. Johnson, Department of Psychology, 404C W. 17th (East Stadium), The Ohio State University, Columbus, OH 43210. however, the needed letter information then would have to be derived from the word-level memorial encoding, rather than being encoded directly.

On the other hand, if the display could not be encoded as a unit, such as would be the case for a meaningless consonant sequence, the initial unsuccessful attempts to encode the pattern as a unit would be followed by attempts to parse and encode the display into memory on an element-by-element basis. However, based on both prior data and logical considerations, it also would be assumed that such element-by-element encoding could occur only after the initial attempts at pattern-level encoding had failed (see Johnson, in press, for a more detailed presentation of these issues and for supporting data).

Given the foregoing, the pattern-unit model would predict that the time to respond to a component of a display always would be delayed by the context of the other components, regardless of whether the pattern was unitizable (e.g., a word) or not unitizable (e.g., a consonant array), and Sloboda (1976) has provided data that support this prediction. However, the delay caused by the context of the other components would be assumed to occur for different reasons, depending upon whether the display was unitizable. In particular, if the array was unitizable, the delay should occur because the entire display had been encoded as a unit and would have to be decoded in order to recover the needed component information, while if the array was not unitizable, the delay should occur because of the initial unsuccessful attempts to provide the array with a unitized representation (see Johnson, Turner-Lyga, \& Pettegrew, 1986, for a more detailed presentation of this distinction and for supporting data).

With regard to the current issue, the major implication of this model is that the initial attempts to treat a display as a unit should result in longer reaction times for detect- 
ing the presence or absence of a predesignated target letter within a consonant array than would be the case if the display consisted of only a single consonant, and that would be true even if the consonant array consisted of several repetitions of the same letter. For example, if subjects were to determine whether a display contained the letter $\mathbf{B}$, and the subsequent display was either a B or an $L$, they would be able to encode the displayed pattern immediately as a unit and compare it to the target. However, if the subsequent display was BBBBB or LLLLL, there should be several initial unsuccessful attempts to encode the pattern as a unit, which would delay the parsing of the display and the encoding of the letter units into memory, and that, in turn, would delay the needed comparison and decision events.

Prior studies (see Johnson, 1977, 1981, for brief descriptions) do offer support for these predictions (see also C. W. Eriksen \& Schultz, 1979, for similar findings). Subjects seemed to be able to detect the presence or absence of a B in single-letter displays (e.g., B or $L$ ) faster than in redundant displays (e.g., BBBBB or LLLLL), but detection was faster in redundant multiletter displays than in nonredundant multiletter displays (e.g., QBTMK or QLTMK). In these studies, the single-letter displays were always presented in a block and the multiletter arrays in another block. Within a block of multiletter displays, half were redundant and half were nonredundant, and they were presented in random order. Furthermore, in one set of conditions subjects were to determine whether the target letter appeared anywhere within the multiletter arrays, while in another set of conditions they were to determine whether the first letter of the multiletter arrays matched the predesignated target.

In the latter conditions the subjects were faster on the redundant displays and the displays in which they were to confine their search to the first-letter position, and the advantage for the redundant arrays was somewhat greater when the target could appear anywhere within the display. In general, then, the reaction times were influenced both by the required search strategy and by the possibility of response competition, as well as by whether the entire presented pattern or only a component of the pattern matched the target.

Although the reaction time advantage for single-letter displays over redundant multiletter displays is consistent with expectations from the pattern-unit model, Krueger and Shapiro (1980) have raised serious questions regarding the reality of the effects, because in a set of very similar experiments, they were not able to obtain the advantage for single-letter displays. In fact, in order to preclude the possibility of experimenter bias, the subjects in one of the Krueger and Shapiro experiments were tested by the same experimenter who had tested the subjects in Johnson's (1977) experiments. Although in my lab she had obtained a substantial advantage for single-letter displays over redundant multiletter arrays, in Krueger's lab she was unable to obtain this effect (the experimenter was unaware of the reason for her testing the subjects in Krueger's lab).

It is true that Krueger and Shapiro's (1980) experiments were not exact replications of Johnson's (1977) work, including the fact that Johnson used black-on-white displays in a tachistoscope, whereas Krueger and Shapiro used a computer-driven CRT (green phosphor),but the experiments were so similar in all the ways that seem important that it is hard not to take the position that their results call into question the reliability of the effect. Accordingly, the goal of the present experiments was to try to reproduce the original effects, as well as to explore them under conditions that approximate more closely those used by Krueger and Shapiro.

Although it comes from a different tradition, and involves a separate collection of empirical issues, the work begun by the Eriksens (B. A. Eriksen \& C. W. Eriksen, 1974) and recently followed up by Grice (see Grice, Boroughs, \& Canham, 1984, and Grice \& Gwynne, 1985, for a review) is relevant to the current issue in that similar kinds of effects have been demonstrated. In the task used in these experiments, subjects are to make one response if one letter appears at their point of fixation and another response if another letter appears, and when the display appears it sometimes has flanking "noise" letters. The present task is similar to the Eriksen-Grice paradigm for the case in which the target and noise letters appear simultaneously.

Although the general result is that presentation of a noise letter identical to the target results in a slower response than when no noise letter is presented (see C. W. Eriksen \& Schultz, 1979; see also Grice \& Gwynne, 1985, for a recent demonstration and review), there is a certain ambiguity regarding this effect as well (e.g., see C. W. Eriksen \& B. A. Eriksen, 1979; Flowers \& Wilcox, 1982). However, it should be noted that there are a number of important procedural and task differences between the Eriksen-Grice paradigm and the task used in the present experiments.

\section{EXPERIMENT 1}

The first experiment was simply a replication of the original study (Johnson, 1977), with the single exception that a different experimenter tested the subjects. In addition, it provided an opportunity to give a detailed report of these effects, which had previously been summarized briefly (Johnson, 1977, 1981). The prediction was that the time to detect whether a display contained a predesignated target letter would be faster if the display consisted of only a single letter than if it contained five repetitions of that letter, but that the reaction time would be faster to such redundant multiletter displays than to nonredundant displays containing five different letters. In addition, for the multiletter displays, if subjects were to confine 
their search to only the first-letter position, the reaction times should be faster than if the target could appear anywhere within the display.

\section{Method}

Subjects. The 30 subjects were introductory psychology students who participated as part of a course option.

Materials. The displays were presented using a Scientific Prototype two-channel tachistoscope. They were typed on a $5 \times 8 \mathrm{in}$. card such that they would appear in the center of the viewing field. The predisplay fixation field consisted of a large mask of Xs that covered the entire viewing field, and there was a dot in the center $\mathrm{X}$ that corresponded to the location of the single-letter display and the middle letter of the multiletter displays. The subjects were fully informed regarding the nature of the displays, and they knew which $\mathrm{Xs}$ in the predisplay fixation field corresponded to the location of the letters in the upcoming target display. The displays were typed in IBM Letter Gothic type (12-point), and each character in a display occupied a viewing angle of $.15^{\circ}$, which means that the fiveletter arrays had a viewing angle of $.75^{\circ}$. The luminance levels were approximately 12 to $14 \mathrm{fL}$.

The displays were presented in blocks of 20 , and each subject was presented with six such blocks. Two of the blocks consisted of single-letter displays ( 20 different uppercase consonants; $\mathrm{Y}$ was not used).

There were two blocks of multiletter displays in which the subjects were to confine their search to the first-letter position (initialposition condition). Half of the displays within a block were redundant and consisted of five repetitions of the same consonant (e.g., BBBBB), while the other half were nonredundant and consisted of five different letters (e.g., BQLTM). Across the 20 displays within a block, 20 different consonants appeared in the initial positions of the displays. The letters used for the redundant displays in one block were used as the initial letters of the nonredundant displays in the other block, and vice versa. No letter occurred more than once within a nonredundant display, and when a target did not appear in the initial position (i.e., was a NO item), it did not appear elsewhere within the display. Within a block, the predesignated target (which was different for each display) matched the initial letter (a YES item) in half the redundant and half the nonredundant displays, and did not appear in the other displays. The consonants that matched the targets (i.e., were YES items) in one block were the NO items for the other block, and vice versa.

The displays in the remaining two blocks also contained five consonants each. Half of the displays were redundant and half were nonredundant. In the nonredundant displays, the target could appear anywhere within the display (any-position condition), and the subjects were fully informed of that fact before those blocks were presented. The structure of the blocks was the same as for the initialposition condition, except that the critical letter appeared in each of the five positions in two displays, and for each subject one of those displays was a YES item and one a NO item. Every display in every block for all of the conditions appeared as a YES item and as a NO item for an equal number of subjects.

Both blocks for a condition were presented together, and each block appeared first and second within a condition for an equal number of subjects. The order in which the three conditions were presented to subjects was counterbalanced so that all 6 possible orders were presented to an equal number of subjects. This resulted in five counterbalancing cycles of six subjects each.

Procedure. When the subjects arrived for the experiment, they were assigned to counterbalanced conditions in a systematic alternating order to ensure that one subject was tested in each of the counterbalancing orders before the next subject was tested in any order. The nature of the displays and of the task was described to each subject before he/she was presented with each block of trials.

Each display was presented for $500 \mathrm{msec}$ (slightly less than the mean reaction time), and the onset of the display started a Hunter timer. Immediately before the onset of the display, the experimenter read the target for that display to the subject, then said "Ready," and the display followed about $500 \mathrm{msec}$ later. The subjects responded by pressing one of two buttons to indicate whether the display did or did not contain the target, and they were allowed to choose which hand to use for the YES response and for the NO response (changing hands was not allowed once testing began). The experimenter recorded the response and the reaction time and then initiated the next display, with approximately 5 to $10 \mathrm{sec}$ between displays, 30 sec between blocks within a condition, and $1 \mathrm{~min}$ between conditions.

\section{Results}

The results are presented in Tables 1 (errors) and 2 (reaction times). An analysis of the data in Table 1 indicated that neither of the main effects nor the interaction was significant. The overall correlation between errors and reaction time across the cells in the two tables is .09; when response type is eliminated as a factor and only the mean for each condition, averaged across response type, is considered, the correlation jumps to .57 . Finally, the correlation between the means in Table 1 and those obtained in the original experiment (Johnson, 1977) is .67. On the whole, then, these error data seem to be similar

Table 1

Error Data (Proportions) From Experiment 1

\begin{tabular}{|c|c|c|c|c|c|}
\hline \multirow{2}{*}{$\begin{array}{c}\text { Response } \\
\text { Type }\end{array}$} & \multirow{2}{*}{$\begin{array}{l}\text { Single- } \\
\text { Letter }\end{array}$} & \multicolumn{2}{|c|}{ Initial-Position } & \multicolumn{2}{|c|}{ Any-Position } \\
\hline & & Redundant & Nonredundant & Redundant & Nonredundant \\
\hline $\begin{array}{l}\text { YES } \\
\text { NO }\end{array}$ & $\begin{array}{l}.027 \\
.027\end{array}$ & $\begin{array}{l}.030 \\
.050\end{array}$ & $\begin{array}{l}.057 \\
.023\end{array}$ & $\begin{array}{l}.037 \\
.027\end{array}$ & $\begin{array}{l}.057 \\
.027\end{array}$ \\
\hline Mean & .027 & .040 & .040 & .032 & .042 \\
\hline
\end{tabular}

Table 2

Latency Data (in Milliseconds) From Experiment 1

\begin{tabular}{|c|c|c|c|c|c|}
\hline \multirow{2}{*}{$\begin{array}{c}\text { Response } \\
\text { Type }\end{array}$} & \multirow{2}{*}{$\begin{array}{l}\text { Single- } \\
\text { Letter }\end{array}$} & \multicolumn{2}{|c|}{ Initial-Position } & \multicolumn{2}{|c|}{ Any-Position } \\
\hline & & Redundant & Nonredundant & Redundant & Nonredundant \\
\hline $\begin{array}{l}\text { YES } \\
\text { NO }\end{array}$ & $\begin{array}{l}548 \\
606\end{array}$ & $\begin{array}{l}614 \\
657\end{array}$ & $\begin{array}{l}631 \\
693\end{array}$ & $\begin{array}{l}667 \\
767\end{array}$ & $\begin{array}{r}894 \\
1,002\end{array}$ \\
\hline Mean & 577 & 635 & 662 & 717 & 948 \\
\hline
\end{tabular}


to those previously obtained, and the positive relationship with reaction time would suggest little problem with speed-accuracy tradeoffs.

The latency data in Table 2 are quite similar to those obtained by Johnson (1977), and this is reflected by the fact that the correlation between the values in the cells within Table 2 and those from the comparable conditions in the 1977 experiment is .83 . An overall analysis of the reaction time data in Table 2 indicated a reliable effect of both conditions $[F(4,116)=72.16, p<.001]$ and response type $[F(1,29)=34.14, p<.001]$, but the interaction was not reliable $[F(4,116)=1.84, p>.05]$. The effect of response type also was reliable for all the specific comparisons described below, and except in cases where it interacted with conditions, it will not be discussed, because this main effect is not relevant to any of the issues at stake in these experiments.

In terms of the specific comparisons, the latency of response to the single-letter displays was less than that to the redundant displays for both the initial-position condition $[F(1,29)=29.24, p<.01]$ and the any-position condition $[F(1,29)=68.21, p<.01]$, and in neither case did the effect interact with response type. In addition, a comparison between the two redundant conditions indicated that subjects were reliably faster for the initialposition condition $[F(1,29)=14.86, p<.01]$ and that effect did interact with response type $[F(1,29)=5.91$, $p<.05]$, with the influence of conditions being somewhat larger on the NO responses.

An overall comparison between the initial-position condition and the any-position condition showed a reliable effect of search condition (i.e., initial-position vs. anyposition) $[F(1,29)=53.02, p<.001]$ and of redundancy $[F(1,29)=99.30, p<.001]$, as well as an interaction between conditions and redundancy $[F(1,29)=48.34$, $p<.001]$. The effect of conditions interacted with response type $[F(1,29)=5.77, p<.05]$. In general, then, the data demonstrate that subjects reacted faster when they knew where to look and the displays were redundant, but the effect of redundancy was somewhat greater when subjects did not know where to look, and not knowing where to look increased the latency for the NO responses more than for the YES responses.

Finally, a specific comparison between the two nonredundant conditions indicated a reliable effect of conditions $[F(1,29)=65.11]$, but this effect did not interact with response type. However, when the only data considered for the YES responses for the any-position condition were those obtained when the target was in the firstletter position $(797 \mathrm{msec})$, the effect of conditions was again reliable $[F(1,29)=45.64, p<.001]$, but in this comparison the effect did interact with response type $[F(1,29)=9.26, p<.01]$.

\section{Discussion}

With respect to the critical issue addressed in this experiment, the correlations between the data in Tables 1 and 2 and comparable values from Johnson's (1977) experiment suggest that the data from these experiments are quite reliable. In addition, the marked latency advantage for the single-letter displays over the redundant displays confirms the original finding in this regard. That would suggest that Krueger and Shapiro's (1980) consistent failure to obtain the effect may rest on a methodological difference between the experiments, rather than its being a basis for questioning the reality of the phenomenon itself.

In terms of the theoretical model on which this work is based, these data are quite consistent with the view that initial attempts to encode small visual patterns are always holistic, inasmuch as they involve attempts to assign a unitary representation to the display as a whole, without any preliminary but analogous attempts to provide comparable cognitive encodings for the display's components. ${ }^{1}$ However, that notwithstanding, Krueger and Shapiro's (1980) data are inconsistent with the model, because the model would predict that a single-letter advantage should be obtained in their experiment as well, but this did not occur. In general, then, although these data reestablish the reliabilty of the advantage of letter detection in singleletter displays over that in redundant displays, the extent to which the effect supports the model from which the prediction arose still seems open to serious question.

A second problem with the interpretation provided by the model is that the single-letter advantage may stem, at least in part, from the type of lateral masking effect demonstrated by Bjork and Murray (1977). Their data indicate that even when response competition is eliminated, as with the redundant displays, letters in close proximity interfere with one another. On the other hand, of course, the interference effect found by Bjork and Murray may have resulted from their subjects' attempting to treat two adjacent letters as if they constituted a single encodable pattern. However, their demonstration that the similarity between the target and the noise item influenced the size of the interference effect suggests that there is more to their data than is captured by that explanation.

With regard to the nature of the search and the effect of redundancy and search condition on response competition, the data are both interesting and quite clear. First, concerning response competition, if nontarget items appear in the array-even in positions that are irrelevant to the decision, as in the initial-position condition-they seem to delay the subject's response. However, when nontarget items appear in potentially relevant positions, as in the any-position condition, the effect is even greater. It could be that these nontarget items signal a competing NO response, with the amount of competition being a function of the relevance of those items, but regardless of relevance the response would be delayed.

The only difficulty with this response-competition interpretation of the redundancy effect is that the redundancy effect was as large, or larger, for the NO responses as for the YES responses, but in the case of a nontarget display there was no competition in that every letter in the array signaled the same negative response. ${ }^{2}$ An alternative interpretation is that the physical regularity of the redundant displays allowed subjects to detect the redun- 
dancy before they began to encode any of the letters. This would signal to the subjects that they could make their decision on the basis of the first letter encoded, rather than waiting until all the letters, or the letter in some specific position, had been encoded, and that advantage should affect the YES and NO responses equally.

If this redundancy-detection strategy was employed infallibly, there always should have been the same redundancy effect, regardless of the context in which the items appeared. However, the data also indicate that subjects were reliably slower in their responses to redundant displays when the necessary search strategy for other items in the list (i.e., the nonredundant items) entailed a scan of the entire display. Probably the simplest interpretation of this apparent spillover of general search strategy to the cases in which a redundant item was presented is that subjects are not perfectly consistent in their use of strategies when they have a task that involves more than one possible strategy.

Finally, with regard to the search strategy, it is clear that knowing where to look for the target within the display is a marked advantage over having to engage in a search. Furthermore, when a search is involved, the earlier in the process the target is encountered, the quicker the response. When the target appeared in the first-letter position the response was significantly faster than when it appeared in one of the later positions within the display. In addition, if the search is self-terminating, and prior data (Johnson, in press; Krueger \& Shapiro, 1980) indicate that to be the case in tasks such as this, then in the initial-position condition the search should be confined to the initial position for both the YES and NO responses. However, in the any-position condition, the target should be detected quite early if it appears in the first-letter position, but if the target does not appear in the display, every position would have to be searched before a response could be made. In general, then, the influence of type of search in this comparison should be greater on the NO responses than on the YES responses, and the reliable search condition $X$ response type interaction supports that expectation $[F(1,29)=9.26, p<.01]$. However, it should be noted that almost all subjects chose to use the right hand for YES and the left hand for NO, and that does confound response type with the hand with which the response was made. However, in that the foregoing issue involves the interaction, and not the main effect of response type, that should not be a problem.

\section{EXPERIMENT 2}

Experiment 2 had two purposes. The first was to reduce, at least to a small degree, the possible influence of lateral masking in this task. This was done by leaving a space between adjacent letters within the multiletter displays, rather than having them immediately adjacent as in the first experiment. This manipulation was not really expected to have much of an influence, because a comparison between spaced and not spaced was included in the original series of experiments (Johnson, 1977), and the advantage for single-letter displays over the redundant displays was greater under those conditions. This result was also obtained in Krueger and Shapiro's (1980) and C. W. Eriksen and B. A. Eriksen's (1979) experiments.

The major purpose of this experiment, however, was based on the apparent influence of the general search strategy for a list on the way the redundant displays were handled. The influence of that strategy does raise a question as to whether the overall disadvantage for the redundant displays in comparison with the single-letter displays may have been the result of a spillover of some strategy unique to the way subjects deal with nonredundant displays, regardless of the nature of the search. This point also was noted by Krueger and Shapiro (1980). In order to eliminate this problem, the redundant and nonredundant displays were segregated into separate lists, and the subjects were thoroughly instructed regarding the nature of a list, and were shown examples, before the display was presented.

\section{Method}

The materials and procedure were exactly the same as for Experiment 1 , with the exceptions that a single space was left between adjacent letters in the displays, and within a search condition the redundant and nonredundant displays were segregated into separate lists. The subjects were 40 students drawn from the same population as was used in Experiment 1.

The counterbalancing scheme included the arbitrary assignment of one of the two redundant lists to each of the two nonredundant lists. Although the nonredundant lists differed in terms of the location of the target items and in the instructions, there were no such differences between the two redundant lists. The reason for the arbitrary assignment of these redundant lists to "conditions" was to allow for the type of conditions $\times$ redundancy analysis performed for Experiment 1.

Half of the subjects saw the single-letter displays before the multiletter displays, and the reverse was true for the other half, and within each of these groups, half of the subjects were given the initialposition lists before the any-position lists, and the reverse was true for the other half. Finally, within each of these subgroups, half of the subjects were given the redundant lists before the nonredundant lists, and the reverse was true for the other half. Thus, the counterbalancing consisted of five cycles of 8 subjects each.

\section{Results}

The results are presented in Tables 3 (errors) and 4 (reaction times). Again, the error data are quite similar to those obtained in the original experiment (Johnson, 1977) $(r=.67)$. The overall analysis indicated that neither the effect of conditions $[F(4,156)=1.86, p>.05]$ nor the effect of response type $[F(1,39)=2.57, p>.05]$ was significant, but the interaction was reliable $[F(4,156)$ $=2.78, p<.05]$. However, the correlation between errors and reaction time was +.21 , and when response type was eliminated as a factor the correlation increased to .67 . Again, then, speed-accuracy tradeoff is not an issue of concern.

The latency data are reported in Table 4. The values across the cells within this table correlate .80 with the corresponding values from the original experiment (Johnson, 1977). An overall analysis of the data from Table 4 
Table 3

Error Data (Proportions) From Experiment 2

\begin{tabular}{|c|c|c|c|c|c|}
\hline \multirow{2}{*}{$\begin{array}{c}\text { Response } \\
\text { Type }\end{array}$} & \multirow{2}{*}{$\begin{array}{l}\text { Single- } \\
\text { Letter }\end{array}$} & \multicolumn{2}{|c|}{ Initial-Position } & \multicolumn{2}{|c|}{ Any-Position } \\
\hline & & Redundant & Nonredundant & Redundant & Nonredundant \\
\hline YES & .024 & .048 & .053 & .028 & .068 \\
\hline NO & .028 & .045 & .030 & .043 & .028 \\
\hline Mean & .026 & .046 & .041 & .035 & .048 \\
\hline
\end{tabular}

Table 4

Latency Data (in Milliseconds) From Experiment 2

\begin{tabular}{ccccccc}
\hline \multirow{2}{*}{$\begin{array}{c}\text { Response } \\
\text { Type }\end{array}$} & Single- & \multicolumn{2}{c}{ Initial-Position } & & \multicolumn{2}{c}{ Any-Position } \\
& Letter & Redundant & Nonredundant & & Redundant & Nonredundant \\
\hline YES & 517 & 559 & 591 & & 533 & 786 \\
NO & 571 & 600 & 639 & & 590 & 906 \\
Mean & 544 & 579 & 615 & & 562 & 846 \\
\hline
\end{tabular}

indicates a significant effect of conditions $[F(4,156)=$ $101.80, p<.001]$ and of response type $[F(1,156)=$ $103.41, p<.0011$, as well as a reliable interaction $[F(4,156)=6.04, p<.01]$. As it was in Experiment 1 , the effect of response type was significant for all of the specific analyses reported below, but because the effect is of no critical significance within these experiments, it will not be discussed except in those instances where it interacts with conditions.

As noted above, the two redundant lists were arbitrarily assigned to conditions, and as expected, an analysis indicated no reliable difference between the means $[F(1,39)=1.51, p>.05]$ and no interaction with response type $(F<1.00)$. Consequently, the two conditions were combined, with the mean YES and NO reaction times being 546 and $595 \mathrm{msec}$, respectively. A comparison of this combined redundant condition with the single-letter displays indicated a significant effect of conditions $[F(1,39)=6.71, p<.05]$, but the interaction with response type was not significant $(F<1.00)$. Therefore, the reaction time advantage of single-letter displays over redundant multiletter displays was again obtained, but it should be noted that the magnitude of the effect was about half that obtained in Experiment 1 and in the original experiments (Johnson, 1977).

A separate analysis comparing the initial-position and any-position conditions, including the redundant as well as nonredundant displays, yielded reliable main effects for search condition $[F(1,39)=79.12, p<.01]$ and for redundancy $[F(1,39)=133.10, p<.001]$, as well as reliable interactions between search condition and redundancy $[F(1,39)=77.87, p<.001]$, search condition and response type $[F(1,39)=7.90, p<.01]$, and redundancy and response type $[F(1,39)=4.20, p<.05]$. The three-way interaction also was significant $[F(1,39)=$ $11.78, p<.01]$. The main difference between these results and those of Experiment 1 is that, for obvious reasons, the difference between the two redundant conditions obtained in the first experiment did not occur in this experiment.

Finally, a comparison between the two nonredundant conditions indicated that the subjects were faster in the initial-position condition $[F(1,39)=111.12, p<.001]$, and this effect interacted with response type $[F(1,39)=$ $19.57, p<.001]$, demonstrating a somewhat larger effect of conditions on the NO responses than on the YES responses. However, again, in order to make the two nonredundant conditions more comparable, an analysis was performed in which the data for the YES responses for the any-position condition were confined to responses obtained when the target appeared in the first-letter position $(672 \mathrm{msec})$. In this analysis, the advantage for the initial-position condition was reliable $[F(1,39)=80.04$, $p<.001]$, as was the condition $\times$ response type interaction $[F(1,39)=38.89, p<.001]$.

\section{Discussion}

As in Experiment 1, the subjects were somewhat faster at responding when asked to detect a target letter when the display consisted of a single letter than when it was a multiletter display. This occurred even when the multiletter displays were redundant (contained five instances of the same letter, e.g., BBBBB). In general, then, the data do seem to be in accord with the predictions of the pattern-unit model. That is, if a subject's initial attempt to deal with a small pattern always is holistic, even when that strategy will eventually fail, the subject should be able to process a single letter more rapidly when it is presented in isolation (i.e., it is the pattern) than when it is presented in the context of other letters (i.e., it is only a component of a larger, ill-defined pattern). This should be true even when the other letters are repetitions of the target letter.

One qualification of these conclusions, however, is that the single-letter advantage in Experiment 2 was substantially smaller than that obtained either in Experiment 1 or in the original experiments (Johnson, 1977). This might be attributable, in part, to the fact that the letters were spaced in this experiment, which could have reduced lateral masking effects. However, Johnson (1977) included one experiment in which between-letter spacing was the only difference between conditions, and not only was the single-letter advantage not reduced, it was actually increased by $10 \mathrm{msec}$, which would argue against the spacing's being the reason for the reduced difference. In 
addition, both Krueger and Shapiro (1980) and C. W. Eriksen and B. A. Eriksen (1979) have reported data suggesting that spacing is irrelevant.

A more likely explanation for the reduced single-letter advantage is the change in the structure of the lists. That is, if part of the originally obtained disadvantage for the redundant displays, in comparison with the single-letter displays, stemmed from the fact that the redundant displays appeared in lists containing nonredundant items, and there was some carryover in subjects' processing strategies from the nonredundant displays to the redundant displays, then the single-letter advantage should have disappeared when lists were homogeneous with respect to redundancy. Although this did not occur, the fact that the effect was reduced suggests that at least part of the original phenomenon may be attributable to a strategic effect.

The possibility of such a strategic carryover is reinforced by the results of Experiment 1 . Those results indicated that the way in which subjects handled the redundant displays was critically influenced by the nature of the search task required by the nonredundant displays in the same list. Given that effect, it does not seem unreasonable that the mere presence of nonredundant items in a list could influence the way in which the redundant items are handled. In fact, of course, just having experienced the nonredundant items during the experiment, even if they were not in the same list as the redundant items, could influence subjects' handling of the redundant items, but this is less likely.

Regarding the effects of redundancy and the search task, the results of Experiment 2 point to the same conclusions as do the results of Experiment 1. The redundancy effect suggests the possibility of response competition, but this explanation would account for the effect only for the YES responses. The fact that the effect is as large, or larger, for the NO items indicates that the most reasonable explanation probably lies elsewhere.

Again, the alternative interpretation seems to be the most appealing. That is, the subjects may detect the regularity of the pattern for the redundant arrays, and thus may know that they can make their decision on the basis of the first letter encoded, regardless of which letter it might be. This interpretation also would be consistent with the fact that in Experiment 1 the magnitude of the redundancy effect (i.e., the difference between the redundant and nonredundant conditions) was related to the amount of subsequent encoding that was avoided.

The data from the NO responses also are consistent with the foregoing account, provided one makes an additional assumption. This assumption is that, as has been argued by Johnson et al. (1986) and Krueger (1970, 1973), NO (or mismatch) responses are always slower than YES (or match) responses because the perceiver of a mismatch display engages in a component-by-component double check of the display before making the negative response (it is assumed that there is no double check for YES responses). Johnson et al. and Krueger have demonstrated that the magnitude of the YES-NO difference is a function of the amount of encoding and comparison that would need to occur after the initial determination of the mismatch in order for the double check to occur. In fact, Johnson et al. (1986) even included a rare case in which the usual effect was reversed (i.e., NO was faster than YES) when the total amount of processing needed to make a negative response, including that needed for the double check, was less than that needed for a YES response.

The larger difference between the YES and NO responses for the nonredundant displays in the present experiment can be explained in two ways. First, because the visual search of consonant arrays appears to be selfterminating, it would be expected that the subjects would have to scan all the letters to know the target is not among them, but they should have to scan only half the display, on average, before finding the target when it is present. The more items to be scanned, the bigger the YES-NO difference, which would mean that the difference would be larger for the nonredundant arrays than for the redundant arrays (which it was). However, because no scan is involved for either the redundant or the nonredundant displays in the initial-position condition, the pattern of higher order interactions should indicate that the interaction of response type with redundancy is limited to the anyposition condition, and the pattern of interactions did so indicate. $^{3}$

Second, if subjects respond immediately when the initial holistic comparison yields a match, but engage in a component-level double check in the case of a mismatch, not only will the NO responses be slower, but the magnitude of that effect should be a function of the amount of double-checking that is necessary. Both in the redundant displays and in the nonredundant displays in the initialposition condition, there would be only one item to double-check, but in the nonredundant displays in the anyposition condition, all five items would be involved. If this type of double check does occur, as previous studies (Johnson et al., 1986; Krueger, 1970, 1973) suggest, in this task it should have a tendency to magnify the YESNO difference for the nonredundant displays compared with the difference for the other four conditions, and the data in Table 4, as well as the pattern of reliable interactions, indicates that this was the case.

The only real problem with this interpretation of the pattern of higher order interactions is that the comparable comparisons in Experiment 1 were not reliable. The critical comparison is between the nonredundant displays in the two search conditions, particularly the interaction with response type, but although that interaction was not significant in Experiment 1, it should be noted that the YES-NO difference for the any-position condition is almost double that for the initial-position condition. It is entirely possible that the strategy ambiguities ensuing from the mixed lists could result in behavioral "noise" which would conceal real differences in the handling of NO responses between the two tasks. The fact that such a large 
mean difference between YES and NO responses from two conditions was not significant is certainly an unusual outcome.

With regard to the nature of the search, the data again are quite consistent with those from Experiment 1. As noted above, when the task required a scan of the entire display (any-position condition), there was an increase in reaction time, and the effect was larger for the NO responses than for the YES responses. In addition, when the only reaction times considered for the YES responses in the any-position condition were those obtained when the target was in the first-letter position, the mean difference between YES and NO responses was almost double that obtained when the mean YES reaction time was based on the mean reaction times for all positions. These data, taken together, again point to a strategy that appears to be a serial self-terminating scan.

\section{CONCLUSIONS}

First, the major purpose of this work was to attempt to reaffirm the reliability of the advantage subjects have when their task is to detect single letters in single-letter displays, as compared with when they appear in displays that consist of multiple repetitions of the same letter. Although a comparison of Experiments 1 and 2 suggests that part of this effect may arise from processing differences that are under the strategic control of the subject (i.e., the effect is reduced when subjects do not know the nature of the display in advance), the results of both experiments did show a reliable advantage for single-letter displays.

However, in terms of the model of holistic processing on which these experiments were based, even though the present data are quite consistent with such a view, the fact that Krueger and Shapiro (1980) failed to obtain a singleletter-display advantage does raise serious questions. There is nothing within the model that can account for their failure to obtain the advantage.

Finally, regarding the nature of the search for the target in tasks of this sort, the data suggest that it is serial and self-terminating, and subjects do seem to be able to confine their search to specific prespecified target locations. One possible interpretation of the redundancy effect is that subjects use the physical regularity of the pattern to detect the redundancy, and the reaction time advantage for redundant displays occurs because the subjects then know that a decision can be made on the basis of the very first detected letter. However, it was noted that when the redundant and nonredundant displays are intermixed within the same list, there may be some carryover of the processing strategy from one display to the next, which would tend to cloud the clarity of the differences in the ways these two types of display are handled.

\section{REFERENCES}

BJork, E. L., \& Murray, J. T. (1977). On the nature of input channels in visual processing. Psychological Review, 84, 472-484.

ERIKSEN, B. A., \& ERIKSEN, C. W. (1974). Effects of noise letters upon the identification of a target letter in a nonsearch task. Perception \& Psychophysics, 16, 143-149.

ERIKSEN, C. W., \& ERIKSEN, B. A. (1979). Target redundancy in visual search: Do repetitions of the target within the display impair processing? Perception \& Psychophysics, 26, 195-205.

ERIKSEN, C. W., \& SChUltz, D. W. (1979). Information processing in visual search: A continuous flow conception and experimental results. Perception \& Psychophysics, 25, 249-263.

Flowers, J. H., \& Wilcox, N. (1982). The effect of flanking context on visual classification: The joint contributions of interactions at different processing levels. Perception \& Psychophysics, 32, 581-591.

Grice, G. R., Boroughs, J. M., \& Canham, L. (1984). Temporal dynamics of associative interference and facilitation produced by visual context. Perception \& Psychophysics, 36, 499-507.

GrICE, G. R., \& GWYNNE, J. W. (1985). Temporal characteristics of noise conditions producing facilitation and interference. Perception \& Psychophysics, 37, 495-501.

JoHnson, N. F. (1975). On the function of letters in word identification: Some data and a preliminary model. Journal of Verbal Learming \& Verbal Behavior, 14, 17-29.

JoHNSON, N. F. (1977). A pattern unit model of word identification. In D. LaBerge \& S. J. Samuels (Eds.), Basic processes in reading. Perception and comprehension. Hillsdale, NJ: Erlbaum.

JoHNSON, N. F. (1981). Integration processes in word recognition. In O. J. Tzeng \& H. Singer (Eds.), Perception of print: Reading research in experimental psychology. Hillsdale, NJ: Erlbaum.

JoHNSON, N. F. (in press). On looking at letters within words: Do we "see" them in memory? Journal of Memory \& Language.

Johnson, N. F., Turner-LygA, M., \& PeTtegrew, B. S. (1986). Partwhole relationships in the processing of small visual patterns. Memory \& Cognition, 14, 5-16.

KRUEGER, L. E. (1970). The effect of bracketing lines on speed of same-different judgements. Journal of Experimental Psychology, 84, 324-330.

KRUEGER, L. E. (1973). Effect of irrelevant surrounding material on speed of same-different judgements of two adjacent letters. Journal of Experimental Psychology, 98, 252-259.

KRUEGER, L. E., \& SHAPIRO, R. G. (1980). Repeating the target neither speeds nor slows its detection: Evidence for independent channels in letter processing. Perception \& Psychophysics, 28, 68-76.

SLoboda, J. A. (1976). Decision times for word and letter search: A wholistic word identification model examined. Journal of Verbal Learning \& Verbal Behavior, 15, 93-101.

SLOBODA, J. A. (1977). The locus of the word-priority effect in a targetdetection task. Memory \& Cognition, 5, 371-376.

YEH, Y.-Y., \& ERIKSEN, C. W. (1984). Name codes and features in the discrimination of letter forms. Perception \& Psychophysics, 36, 225-233.

\section{NOTES}

1. See Krueger and Shapiro (1980), Yeh and C. W. Eriksen (1984), and Grice and Gwynne (1985) for a very different type of theoretical treatment of these issues.

2. It should be noted that the noninitial letters in the nonredundant displays for the initial-position condition really approximate the irrelevant noise items, rather than the incompatible noise items, in the EriksenGrice paradigm.

3. Again, it should be remembered that the hands subjects used for the YES and NO responses were not counterbalanced, and consequently any differences between YES and NO responses could be attributed to response execution factors. However, all of the foregoing and following considerations that are critical involve the way in which response type interacted with the other variables, not the main effect, and there is no reason to suppose that the interactions would be contaminated by the failure to counterbalance. 